\title{
Allergieforscher haben die Fachgutachterwahl
}

\author{
Bei der DFG wird es 2012 erstmals ein Fachkollegium Allergologie \\ geben. Gemeinsam mit Rheumatologie und klinischer Immunologie \\ wird es Forschungsanträge mit allergologischen Inhalten begutachten. \\ Wie es sich zusammensetzt, können Sie aktiv mitentscheiden: in der \\ Fach gutachterwahl vom 7. November bis 5. Dezember 2011.
}

\begin{abstract}
_ ür eine faire Begutachtung von Forschungsanträgen mit allergologischem Inhalt bei der Deutschen Forschungsgemeinschaft (DFG) ist es essenziell, dass die Fachgutachter eine hohe Expertise auf dem Gebiet der Allergologie aufweisen. Bisher ist dieses Gebiet nicht durch ein eigenständiges Fachkollegium bei der DFG vertreten, trotz einer deutlichen Zunahme an allergologischen Forschungsanträgen. Um die Situation zu verbessern, hat der Vorstand der Deutschen Gesellschaft für
\end{abstract}

Allergologie und klinische Immunologie (DGAKI) sich in den letzten Jahren intensiv dafür stark gemacht, die allergologische Kompetenz in den entsprechenden Gutachterkreisen zu verstärken bzw. zu erhöhen.

Ein erster großer Erfolg in dieser Richtung ist die Einführung eines eigenständigen Fachkollegiums - zusammen mit der Rheumatologie und der klinischen Immunologie - ab der nächsten Wahlperiode. Diesen Schritt begrüßen wir sehr, können ihn allerdings nur als

Hinweise zum Ablauf der Wahl

Die Frist zur Online-Wahl der Mitglieder der DFG-Fachkollegien für die Amtsperiode 2012 bis 2015 beginnt am 7. November 2011. Wahlberechtigt sind alle Personen, die am ersten Tag der Wahlfrist entweder Professor oder Juniorprofessor sind oder die vor dem ersten Tag der Wahlfrist erfolgreich die mündliche Doktorprüfung abgelegt haben und einer wissenschaftlich forschenden Tätigkeit nachgehen - also auch drittmittelbeschäftigte Wissenschaftler.

Die Wahlberechtigten wurden bis September 2011 durch die wissenschaftliche Einrichtung, an der sie tätig sind, erfasst. Ab Mitte Oktober erhalten sie ihre Wahlunterlagen durch diese Einrichtung

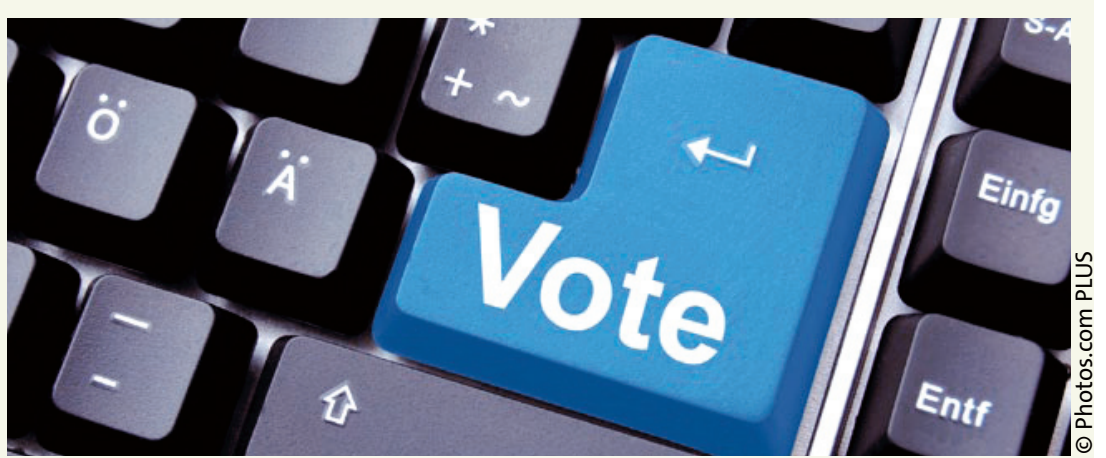

\section{Die Kandidaten der DGAKI}

Die folgenden, von der DGAKI vorgeschlagenen und in der DGAKI ganz aktiven Kolleginnen und Kollegen kandidieren bei der DFG-Wahl.

Fachkollegium 205-13 (Pneumologie) - Prof. Dr. Christian Virchow, Rostock _ Prof. Dr. Christian Taube, Mainz Von acht Kandidaten sind drei zu wählen.

\section{Fachkollegium 205-18 (Allergologie,} Rheumatologie, klinische Immunologie) —-Prof. Dr. Harald Renz, Marburg Von neun Kandidaten sind vier zu wählen.

\section{Fachkollegium 205-19 (Dermatologie) _-Prof. Dr. Tilo Biedermann, Tübingen - Prof. Dr. Thomas Werfel, Hannover Von zehn Kandidaten können vier gewählt werden.}

einen ersten Schritt in die richtige Richtung werten.

Ende dieses Jahres finden die nächsten Fachgutachterwahlen statt. Die Fachgutachter werden für eine Periode von drei Jahren gewählt, sind also über einen längeren Zeitraum hin aktiv. Damit kommt dieser Wahl eine besondere Bedeutung zu.

Im Vorfeld hat die DFG verschiedene medizinisch-wissenschaftliche Fachgesellschaften aufgerufen, Kandidaten für die Wahl zu nominieren. Insgesamt sind bei der DFG 1.841 Vorschläge eingegangen, u.a. auch acht Vorschläge aus dem Kreis der DGAKI. Hieraus hat die DFG insgesamt 1.385 Kandidaten für die 606 zu vergebenden Sitze in verschiedenen Fachkollegien vorselektiert. Aus diesen fast 1.400 Kandidaten wird Ende des Jahres gewählt!

Daher die Bitte des DGAKI-Vorstands: Nehmen Sie an der Wahl teil und motivieren Sie auch alle Kollegen in Ihrem Umfeld, vom Wahlrecht Gebrauch zu machen! Häufeln Sie Ihre Stimmen dabei auf die Kandidaten der DGAKI. Nur so hat die Gesellschaft eine Mindestchance zur adäquaten Repräsentation von Fach und Mitgliedern. Alles in allem ist die Allergologie schon einen Schritt in die richtige Richtung vorangekommen, aber die Ziele sind noch lange nicht erreicht!

Prof. Dr. Harald Renz, Marburg 